\title{
ON THE RIGIDITY OF CERTAIN PHAM-BRIESKORN RINGS
}

\author{
MICHAEL CHITAYAT AND DANIEL DAIGLE
}

\begin{abstract}
Fix a field $\mathbf{k}$ of characteristic zero. If $a_{1}, \ldots, a_{n}(n \geq 3)$ are positive integers, the integral domain $B_{a_{1}, \ldots, a_{n}}=\mathbf{k}\left[X_{1}, \ldots, X_{n}\right] /\left\langle X_{1}^{a_{1}}+\cdots+X_{n}^{a_{n}}\right\rangle$ is called a Pham-Brieskorn ring. It is conjectured that if $a_{i} \geq 2$ for all $i$ and $a_{i}=2$ for at most one $i$, then $B_{a_{1}, \ldots, a_{n}}$ is rigid. (A ring $B$ is said to be rigid if the only locally nilpotent derivation $D: B \rightarrow B$ is the zero derivation.) We give partial results towards the conjecture.
\end{abstract}

\section{INTRODUCTION}

If $B$ is a commutative ring of characteristic zero, a derivation $D: B \rightarrow B$ is locally nilpotent if for each $x \in B$ there exists $n>0$ such that $D^{n}(x)=0$. If the only locally nilpotent derivation $D: B \rightarrow B$ is the zero derivation, one says that $B$ is rigid. One says that $B$ is stably rigid if for any $N \geq 0$ and any locally nilpotent derivation $D: B\left[X_{1}, \ldots, X_{N}\right] \rightarrow B\left[X_{1}, \ldots, X_{N}\right]$ (where $B\left[X_{1}, \ldots, X_{N}\right]$ is the polynomial ring in $N$ variables over $B$ ), we have $D(B)=\{0\}$. Note that stable rigidity implies rigidity.

Fix a field $\mathbf{k}$ of characteristic zero. If $a_{1}, \ldots, a_{n}(n \geq 3)$ are positive integers, the integral domain $B_{a_{1}, \ldots, a_{n}}=\mathbf{k}\left[X_{1}, \ldots, X_{n}\right] /\left\langle X_{1}^{a_{1}}+\cdots+X_{n}^{a_{n}}\right\rangle$ is called a Pham-Brieskorn ring. These rings and the corresponding varieties have been studied extensively and from several angles; paper [10] refers to [13] for a survey. It is interesting to ask which Pham-Brieskorn rings are rigid or stably rigid. Consider the set

$$
T_{n}=\left\{\left(a_{1}, \ldots, a_{n}\right) \in \mathbb{Z}^{n} \mid a_{i} \geq 2 \text { for all } i \text { and } a_{i}=2 \text { for at most one } i\right\} .
$$

It is known (and easy to see) that if $B_{a_{1}, \ldots, a_{n}}$ is rigid then $\left(a_{1}, \ldots, a_{n}\right) \in T_{n}$; so one wants to know if the converse is true. The case $n=3$ is settled by:

1.1. Theorem. Let $a, b, c$ be positive integers.

(a) If $(a, b, c) \in T_{3}$ then $B_{a, b, c}$ is rigid.

(b) If $\frac{1}{a}+\frac{1}{b}+\frac{1}{c} \leq 1$ then $B_{a, b, c}$ is stably rigid.

This is [10, Theorem 7.1] (the case $\mathbf{k}=\mathbb{C}$, with part (b) implicit, is [12, Lemma 4]).

For arbitrary $n \geq 3$, one has:

1.2. Theorem. If $\sum_{i=1}^{n} \frac{1}{a_{i}} \leq \frac{1}{n-2}$ then $B_{a_{1}, \ldots, a_{n}}$ is rigid.

The case $\mathbf{k}=\mathbb{C}$ of Theorem 1.2 is [9, Example 2.6]. The general case follows, because (as one can see) rigidity over $\mathbb{C}$ implies rigidity over any field of characteristic zero.

In the case $n=4$, the following is known:

1.3. Theorem. Assume that $(a, b, c, d) \in T_{4}$. Then $B_{a, b, c, d}$ is rigid in each of the following cases:

2010 Mathematics Subject Classification. Primary: 13N15, 14R20, 14 R05.

Key words and phrases. Locally nilpotent derivations, rigid rings, Pham-Brieskorn varieties, affine varieties, ruled varieties.

Research of both authors supported by grant 04539/RGPIN/2015 from NSERC Canada. 
(a) $\frac{1}{a}+\frac{1}{b}+\frac{1}{c}+\frac{1}{d} \leq \frac{1}{2}$

(b) $\operatorname{gcd}(a b c, d)=1$

(c) $a=b=c=3$

(d) $a=2, b, c, d \geq 3, b$ is even, $\operatorname{gcd}(b, c) \geq 3$ and $\operatorname{gcd}(d, \operatorname{lcm}(b, c))=2$

(e) $\operatorname{cotype}(a, b, c, d) \geq 2$.

Part (e) of Theorem 1.3 is Theorem 7.2(b) of [5], reformulated in terms of cotype (see Definition 3.9 for the notion of cotype). Part (a) is the case $n=4$ of Theorem 1.2. Parts (b-d) are stated in [5, Theorem 7.1] but are proved in [10, Theorem 8.1] and [1, Corollary 1.9].

This article settles many cases not covered by Theorems 1.11.3. In order to avoid giving too many definitions in the Introduction, we only present a subset of our results as Theorem 1.4. Other significant results appear in Section 4. The Pham-Brieskorn rings $B_{a_{1}, \ldots, a_{n}}$ that appear in Theorem 1.4 are defined over an arbitrary field $\mathbf{k}$ of characteristic zero, and we always assume that $n \geq 3$.

\subsection{Theorem.}

(a) If $a \geq n \geq 4$ then $B_{\underbrace{a, \ldots, a}_{n}}$ is rigid.

(b) If $\sum_{i=1}^{n} \frac{1}{a_{i}} \leq \frac{1}{n-2}$ then $B_{a_{1}, \ldots, a_{n}}$ is stably rigid.

(c) If $\sum_{i \in I} \frac{1}{a_{i}}<\frac{1}{n-2}$ then $B_{a_{1}, \ldots, a_{n}}$ is rigid, where $I=\left\{i \mid a_{i}\right.$ divides $\left.\operatorname{lcm}\left(a_{1}, \ldots \widehat{a_{i}} \ldots, a_{n}\right)\right\}$.

(d) If $a, b, c, d \geq 1$ satisfy $a \nmid \operatorname{lcm}(b, c, d)$ and $\frac{1}{b}+\frac{1}{c}+\frac{1}{d}<\frac{1}{2}$ then $B_{a, b, c, d}$ is rigid.

(e) If $\left(a_{1}, \ldots, a_{n}\right) \in T_{n}$ and cotype $\left(a_{1}, \ldots, a_{n}\right) \geq n-2$, then $B_{a_{1}, \ldots, a_{n}}$ is rigid.

(f) If $k_{1}, k_{2}, k_{3}, k_{4} \geq 1$ are pairwise relatively prime and $a \geq 3$ then $B_{a k_{1}, a k_{2}, a k_{3}, a k_{4}}$ is rigid.

(g) If $k_{1}, \ldots, k_{n} \geq 1$ are pairwise relatively prime and $a \geq n \geq 4$ then $B_{a k_{1}, \ldots, a k_{n}}$ is rigid.

(h) If $a_{1}, \ldots, a_{m} \geq 1(m \geq 1)$ satisfy $a_{i} \nmid \operatorname{lcm}\left(3, a_{1}, \ldots \widehat{a_{i}} \ldots, a_{m}\right)$ for all $i \in\{1, \ldots, m\}$, then $B_{a_{1}, \ldots, a_{m}, 3,3,3}$ is rigid.

Part (a) (of Theorem 1.4) is Corollary 4.6, the fact that $B_{a, \ldots, a}$ is rigid when $n \leq a<n(n-2)$ appears to be a new result (if $a \geq n(n-2)$ then $B_{a, \ldots, a}$ is rigid by Theorem 1.2). Parts (b) and (c) are Corollary 6.8 and Proposition 4.12, respectively; these two results strengthen Theorem 1.2 , Part (d) is the case " $n=4$ " of part (c). Part (e) is Corollary 4.16, it generalizes Theorem 1.3(e). Parts (f) and (g) are Corollary 4.10, and $(\mathrm{h})$ is Example 4.17, parts $(\mathrm{f}-\mathrm{h})$ are illustrations of stronger results that cannot be stated in the Introduction.

Let us say a few words about Section 3. It is known that if a $\mathbf{k}$-domain $B$ is not rigid then its field of fractions $\operatorname{Frac}(B)$ is ruled over $\mathbf{k}$, i.e., there exists a field $K$ such that $\mathbf{k} \subseteq K \subset \operatorname{Frac}(B)$ and $\operatorname{Frac}(B)=K(t)$ where $t$ is transcendental over $K$. So, one technique for showing that $B$ is rigid is to show that $\operatorname{Frac}(B)$ is not ruled over $\mathbf{k}$. However, that technique is useless when $B$ admits a nontrivial $\mathbb{Z}$-grading, because then $\operatorname{Frac}(B)$ is always ruled over k: we have $\operatorname{Frac}(B)=K(t)$ where $K=\operatorname{HFrac}(B)$ is the "homogeneous field of fractions" of $B$. Section 3 shows that if $B$ is a graded k-domain which is not rigid then, under certain additional hypotheses, HFrac $(B)$ itself is ruled over $\mathbf{k}$. So, to prove that a graded domain $B$ satisfying certain assumptions is rigid, it suffices to show that $\operatorname{HFrac}(B)$ is not ruled. It is this technique that allows us to prove part (a) of Theorem 1.4 , 


\section{Preliminaries}

Throughout this work, all rings are commutative and have a multiplicative identity 1 . All ring homomorphisms map 1 to 1 . If $B$ is a ring then $B^{*}$ denotes its group of units. If $b \in B$ then $\langle b\rangle$ is the ideal of $B$ generated by $b$, and we use the notation $B_{b}=S^{-1} B$ where $S=\left\{1, b, b^{2}, \ldots\right\}$. By a domain, we mean an integral domain. If $B$ is a domain, its fraction field is denoted $\operatorname{Frac}(B)$. If $A \subseteq B$ are domains then $\operatorname{trdeg}_{A}(B)$ denotes the transcendence degree of $\operatorname{Frac}(B)$ over $\operatorname{Frac}(A)$. A subring $A$ of a domain $B$ is factorially closed in $B$ if for all $x, y \in B \backslash\{0\}$ we have the implication $x y \in A \Rightarrow x, y \in A$. If $\mathbf{k}$ is a field, then a $\mathbf{k}$-domain is a domain that is also a $\mathbf{k}$-algebra. An affine $\mathbf{k}$-domain is a $\mathbf{k}$-domain that is finitely generated as a $\mathbf{k}$-algebra.

If $A$ is a subring of a ring $B$, we write $B=A^{[n]}$ to indicate that $B$ is isomorphic to the polynomial algebra in $n$ variables over $A$. If $K / \mathbf{k}$ is a field extension, $K=\mathbf{k}^{(n)}$ means that $K$ is purely transcendental over $\mathbf{k}$, of transcendence degree $n$.

We write " $\subseteq$ " for inclusion and " $\subset$ " for proper inclusion.

Let $B$ be a ring and $D: B \rightarrow B$ a derivation. We say that $D$ is irreducible if the only principal ideal of $B$ containing $D(B)$ is $B$ itself. We say that $D$ is locally nilpotent if for each $b \in B$ there exists an $n \in \mathbb{N}$ such that $D^{n}(b)=0$. A slice of $D$ is an element $t \in B$ such that $D(t)=1$. A preslice of $D$ is an element $t \in B$ such that $D(t) \neq 0$ and $D^{2}(t)=0$.

2.1. Definition. If $B$ is a ring, the set of locally nilpotent derivations $D: B \rightarrow B$ is denoted $\operatorname{LND}(B)$. If $\operatorname{LND}(B)=\{0\}$, we say that $B$ is rigid.

2.2. Let $B$ be an integral domain of characteristic zero, let $D: B \rightarrow B$ be a derivation, and let $A=\operatorname{ker} D$. The following facts are well known (refer to [8], [1] or [3]).

(a) If $D$ is locally nilpotent, then $A$ is a factorially closed subring of $B$. Consequently, if $D$ is locally nilpotent and $\mathbf{k}$ is a field included in $B$ then $D$ is a $\mathbf{k}$-derivation.

(b) Assume that $\mathbb{Q} \subseteq B$. If $D \neq 0$ is locally nilpotent then $D$ has a preslice $t \in B$. For any such $t$, if we define $\alpha=D(t)$ then $B_{\alpha}=A_{\alpha}[t]=\left(A_{\alpha}\right)^{[1]}$. Consequently, $\operatorname{trdeg}_{A}(B)=1$ and $\operatorname{Frac}(B)=(\operatorname{Frac}(A))^{(1)}$.

(c) If $D \neq 0$ is locally nilpotent and $B$ satisfies the ascending chain condition for principal ideals, then there exists an irreducible locally nilpotent derivation $\delta: B \rightarrow B$ such that $D=a \delta$ for some $a \in A$.

(d) Let $S \subseteq B \backslash\{0\}$ be a multiplicative subset of $B$ containing 1 . Then $S^{-1} D: S^{-1} B \rightarrow S^{-1} B$ defined by $\left(S^{-1} D\right)\left(\frac{b}{s}\right)=\frac{s D(b)-b D(s)}{s^{2}}$ is a derivation and the following hold:

(i) $S^{-1} D$ is locally nilpotent if and only if $D$ is locally nilpotent and $S \subseteq A$;

(ii) if $S \subseteq A$ then $\operatorname{ker} S^{-1} D=S^{-1} A$ and $S^{-1} A \cap B=A$.

2.3. Definition. Let $B$ be a ring of characteristic zero. If $D \in \operatorname{LND}(B)$ then define $\operatorname{deg}_{D}: B \rightarrow$ $\mathbb{N} \cup\{-\infty\}$ by declaring that $\operatorname{deg}_{D}(0)=-\infty$ and that $\operatorname{deg}_{D}(x)=\max \left\{n \in \mathbb{N} \mid D^{n}(x) \neq 0\right\}$ for each $x \in B \backslash\{0\}$. Also define $|x|_{B}=\min \left\{\operatorname{deg}_{D}(x) \mid D \in \operatorname{LND}(B) \backslash\{0\}\right\}$ for each $x \in B \backslash\{0\}$, where we adopt the convention that $\min \varnothing=\infty$, so $|x|_{B}=\infty$ when $B$ is rigid.

\section{Derivations of G-GRADED RINGS}

3.1. Definition. Let $(G,+)$ be an abelian group. A $G$-grading of a ring $B$ is a family $\left\{B_{g}\right\}_{g \in G}$ of subgroups of $(B,+)$ such that $B=\bigoplus_{g \in G} B_{g}$ and $B_{g} B_{h} \subseteq B_{g+h}$ for all $g, h \in G$. A $G$-graded ring is a ring $B$ together with a $G$-grading (of $B$ ). In the special case where $G=\mathbb{Z}$ and $B_{i}=0$ for all $i<0$, we say that $B$ is $\mathbb{N}$-graded and write $B=\bigoplus_{i \in \mathbb{N}} B_{i}$. 
3.2. Definitions. Suppose that $G$ is an abelian group and that $B=\bigoplus_{i \in G} B_{i}$ is a $G$-graded ring.

- A derivation $D: B \rightarrow B$ is homogeneous if there exists an $h \in G$ such that $D\left(B_{g}\right) \subseteq B_{g+h}$ for all $g \in G$. If $D$ is homogeneous and $D \neq 0$ then $h$ is unique and we say that $D$ is homogeneous of degree $h$. The zero derivation is homogeneous of degree $-\infty$. The set of homogeneous locally nilpotent derivations of $B$ is denoted $\operatorname{HLND}(B)$.

- A graded subring of $B$ is a subring $A$ of $B$ satisfying $A=\bigoplus_{g \in G}\left(A \cap B_{g}\right)$. If $A$ is a graded subring of $B$ then $A$ is a $G$-graded ring $\left(A=\bigoplus_{g \in G} A_{g}\right.$ is a $G$-grading of $A$, where we define $A_{g}=A \cap B_{g}$ for each $\left.g \in G\right)$. Note that if $D \in \operatorname{HLND}(B)$ then $\operatorname{ker} D$ is a graded subring of $B$.

- If $A$ is a graded subring of $B$, define $G(A)$ to be the subgroup of $G$ generated by the set $\left\{g \in G \mid A_{g} \neq 0\right\}$.

3.3. Definition. Suppose that $G$ is an abelian group and that $B=\bigoplus_{i \in G} B_{i}$ is a $G$-graded integral domain. If $S$ is a multiplicatively closed subset of $\bigcup_{i \in G}\left(B_{i} \backslash\{0\}\right)$ such that $1 \in S$ then the localized ring $S^{-1} B$ is a $G$-graded ring in a natural way, and if we write $S^{-1} B=R=\bigoplus_{i \in G} R_{i}$ then the subring $R_{0}$ of $S^{-1} B$ called the homogeneous localization of $B$ at $S$. Explicitly,

$$
R_{0}=\bigcup_{i \in G}\left\{\frac{b}{s} \mid b \in B_{i} \text { and } s \in B_{i} \cap S\right\} .
$$

We define $\operatorname{HFrac}(B)$ to be the homogeneous localization of $B$ at $S=\bigcup_{i \in G}\left(B_{i} \backslash\{0\}\right)$. It is clear that $\operatorname{HFrac}(B)$ is a subfield of $\operatorname{Frac}(B)$; we call $\operatorname{HFrac}(B)$ the homogeneous field of fractions of $B$.

3.4. Example. If $B$ is an $\mathbb{N}$-graded integral domain such that $B \neq B_{0}$ then Proj $B$ and Spec $B$ are integral schemes, $\operatorname{Frac}(B)$ is the function field of $\operatorname{Spec} B, \operatorname{HFrac}(B)$ is the function field of $\operatorname{Proj} B$, and $\operatorname{Frac}(B)=(\operatorname{HFrac}(B))^{(1)}$. (If $B=B_{0}$ then $\operatorname{Proj} B=\varnothing$ is not an integral scheme and hence does not have a function field. Note that $\operatorname{Frac}(B)=\operatorname{HFrac}(B)$ whenever $B=B_{0}$.)

3.5. Definition. A field extension $L / \mathbf{k}$ is ruled if there exists a field $K$ such that $\mathbf{k} \leq K \leq L$ and $L=K^{(1)}$.

3.6. Theorem. Let $G$ be an abelian group and $B$ a $G$-graded integral domain of characteristic zero. Let $D \in \operatorname{HLND}(B) \backslash\{0\}$, let $A=\operatorname{ker} D$ and suppose that $G(A)=G(B)$.

(a) $\operatorname{HFrac}(B)=(\operatorname{HFrac}(A))^{(1)}$

(b) If $\mathbf{k}$ is a field included in $B$ then $\mathbf{k} \cap B_{0}$ is a field included in $\operatorname{HFrac}(A)$.

(c) If $G$ is torsion-free and $\mathbf{k}$ is a field included in $B$ then $\mathbf{k} \subseteq \operatorname{HFrac}(A)$ and consequently $\operatorname{HFrac}(B)$ is ruled over $\mathbf{k}$.

Proof. If $B=B_{0}$ then $\operatorname{Frac}(B)=\operatorname{HFrac}(B)$ and $\operatorname{Frac}(A)=\operatorname{HFrac}(A)$, so the Theorem follows from parts (国) and (b) of 2.2. So we may assume that $B \neq B_{0}$.

(a) Let $d=\operatorname{deg} D$ and $S=\bigcup_{g \in G}\left(A_{g} \backslash\{0\}\right)$. Then $S^{-1} D: S^{-1} B \rightarrow S^{-1} B$ is nonzero and locally nilpotent, and also homogeneous of degree $d$. Since $-d \in G(B)$ and $G(A)=G(B)$, there exist $a, s \in S$ such that the homogeneous element $\frac{a}{s} \in S^{-1} A$ has degree $-d$. Then $\frac{a}{s} S^{-1} D$ : $S^{-1} B \rightarrow S^{-1} B$ is a homogeneous derivation of degree 0 ; since $\frac{a}{s} \in \operatorname{ker}\left(S^{-1} D\right)$ and $S^{-1} D$ is locally nilpotent, $\frac{a}{s} S^{-1} D$ is locally nilpotent. Let $B_{(S)}$ denote the degree 0 subring of $S^{-1} B$ and let $D_{(S)}: B_{(S)} \rightarrow B_{(S)}$ be the restriction of $\frac{a}{s} S^{-1} D$. Then $D_{(S)}$ is a locally nilpotent derivation whose kernel is $B_{(S)} \cap \operatorname{ker}\left(\frac{a}{s} S^{-1} D\right)=B_{(S)} \cap S^{-1} A=A_{(S)}=\operatorname{HFrac}(A)$. 
We show that $D_{(S)}$ is nonzero. It is straightforward to verify that $D$ has a homogeneous preslice $t \in B$. Since $G(A)=G(B)$, there exist $a^{\prime}, s^{\prime} \in S$ such that $\operatorname{deg}\left(\frac{a^{\prime}}{s^{\prime}}\right)=-\operatorname{deg}(t)$. Then $\frac{a^{\prime} t}{s^{\prime}} \in B_{(S)}$ and, since $\frac{a^{\prime}}{s^{\prime}} \in \operatorname{ker} S^{-1} D, D_{(S)}\left(\frac{a^{\prime} t}{s^{\prime}}\right)=\left(\frac{a}{s} S^{-1} D\right)\left(\frac{a^{\prime} t}{s^{\prime}}\right)=\frac{a^{\prime}}{s^{\prime}} \frac{a}{s} S^{-1} D(t) \neq 0$.

Since $D_{(S)} \neq 0$ and $\operatorname{ker} D_{(S)}=\operatorname{HFrac}(A)$ is a field, 2.2(b) implies that $B_{(S)}=(\operatorname{HFrac}(A))^{[1]}$. Using $G(A)=G(B)$ once again, we see that $\operatorname{HFrac}(B)$ is the field of fractions of $B_{(S)}$; so $\operatorname{HFrac}(B)=(\operatorname{HFrac}(A))^{(1)}$. This proves (a).

(b) If $\mathbf{k}$ is a field included in $B$ then it is clear that $\mathbf{k} \cap B_{0}$ is a field and 2.2(国) implies that $\mathbf{k} \subseteq A$, so $\mathbf{k} \cap B_{0} \subseteq A \cap B_{0}=A_{0} \subseteq \operatorname{HFrac}(A)$.

(c) It is well known that if $G$ is a torsion-free abelian group and $B$ is a $G$-graded domain then any field included in $B$ is in fact included in $B_{0}$ (see [2, Lemma 2.4.7], for instance). So in the present situation we have $\mathbf{k} \subseteq B_{0}$; then (b) implies that $\mathbf{k} \subseteq \operatorname{HFrac}(A)$ and (a) implies that $\operatorname{HFrac}(B)$ is ruled over $\mathbf{k}$.

3.7. Remark. In the special case where the grading is an $\mathbb{N}$-grading and is nontrivial $\left(B \neq B_{0}\right)$, Theorem 3.6(c) asserts that the function field of Proj $B$ is ruled over k. (See Example 3.4.) The same remark applies to Proposition 3.10, below.

3.8 (Homogenization). Let $\mathbf{k}$ be a field of characteristic zero and $B=\bigoplus_{i \in \mathbb{Z}} B_{i}$ a $\mathbb{Z}$-graded affine k-domain. One can show that if $D \in \operatorname{LND}(B)$ then the subset $S_{D} \stackrel{\text { def }}{=}\{\operatorname{deg}(D x)-\operatorname{deg}(x) \mid$ $x \in B \backslash\{0\}\}$ of $\mathbb{Z} \cup\{-\infty\}$ has a greatest element (where deg : $B \rightarrow \mathbb{Z} \cup\{-\infty\}$ is the degree function determined by the grading of $B$ ); one defines $\operatorname{deg}(D)=\max \left(S_{D}\right) \in \mathbb{Z} \cup\{-\infty\}$. Clearly, $\operatorname{deg}(D)=-\infty \Leftrightarrow D=0$. Also, if $D$ happens to be homogeneous then $\operatorname{deg}(D)$ coincides with the usual degree of a homogeneous derivation (Definition 3.2).

If $D \in \operatorname{LND}(B)$ then there is a natural way to define an element $\tilde{D}$ of $\operatorname{HLND}(B)$ satisfying (in particular) $\operatorname{deg}(\tilde{D})=\operatorname{deg}(D)$. Indeed, if $D=0$ then set $\tilde{D}=0$. If $D \in \operatorname{LND}(B) \backslash\{0\}$ then let $d=\operatorname{deg}(D) \in \mathbb{Z}$ and define (for each $i \in \mathbb{Z}$ ) a map $\tilde{D}_{i}: B_{i} \rightarrow B_{i+d}$ by $\tilde{D}_{i}(x)=p_{i+d}(D x)$, where $p_{j}: B \rightarrow B_{j}$ is the canonical projection; then if $b=\sum_{i \in \mathbb{Z}} b_{i} \in B\left(b_{i} \in B_{i}\right.$ for all $i$, $b_{i}=0$ for almost all $i$ ), define $\tilde{D}(b)=\sum_{i} \tilde{D}_{i}\left(b_{i}\right)$; one can check that $\tilde{D} \in \operatorname{HLND}(B) \backslash\{0\}$ and $\operatorname{deg}(\tilde{D})=\operatorname{deg}(D)$. Note in particular that $D \neq 0 \Longrightarrow \tilde{D} \neq 0$. The derivation $\tilde{D}$ is sometimes called the homogenization of $D$. This is in fact a special case of the process of replacing $D: B \rightarrow B$ by $\operatorname{Gr}(D): \operatorname{Gr}(B) \rightarrow \mathbf{G r}(B)$, so one can also call $\tilde{D}$ the associated homogeneous derivation of $D$. Note the following consequence of the above discussion:

Let $\mathbf{k}$ be a field of characteristic zero and $B$ a $\mathbb{Z}$-graded affine $\mathbf{k}$-domain. If $B$ is not rigid then $\operatorname{HLND}(B) \neq\{0\}$.

Refer to [4] for proofs of the claims made in 3.8, and for an in-depth treatment of this topic.

3.9. Definitions. Let $n \geq 2$ and $S=\left(a_{1}, a_{2}, \ldots, a_{n}\right) \in \mathbb{Z}^{n}$.

- Define $\operatorname{gcd}(S)=\operatorname{gcd}\left(a_{1}, \ldots, a_{n}\right)$ and $\operatorname{lcm}(S)=\operatorname{lcm}\left(a_{1}, \ldots, a_{n}\right)$.

- If $\operatorname{gcd}(S)=1$, we say that $S$ is normal. If $S \neq(0, \ldots, 0)$ then the tuple $S^{\prime}=\left(\frac{a_{1}}{d}, \ldots, \frac{a_{n}}{d}\right)$ (where $d=\operatorname{gcd}(S)$ ) is normal, and is called the normalization of $S$.

- For each $j \in\{1, \ldots, n\}$, define $S_{j}=\left(a_{1}, \ldots \widehat{a_{j}} \ldots, a_{n}\right)$ ( $j$-th component omitted). More generally, given a proper subset $J$ of $\{1, \ldots, n\}$ we define $S_{J}=\left(a_{i_{1}}, \ldots, a_{i_{s}}\right)$, where $i_{1}<\cdots<i_{s}$ are the elements of $\{1, \ldots, n\} \backslash J$.

\footnotetext{
${ }^{1}$ By convention, $\operatorname{gcd}(S) \geq 0$ and $\operatorname{lcm}(S) \geq 0$.
} 
- We define the sets

$$
\begin{aligned}
J^{*}(S) & =\left\{i \in\{1, \ldots, n\} \mid \operatorname{gcd}\left(S_{i}\right) \neq \operatorname{gcd}(S)\right\}=\left\{i \in\{1, \ldots, n\} \mid \operatorname{gcd}\left(S_{i}\right) \nmid a_{i}\right\} \\
J(S) & =\left\{i \in\{1, \ldots, n\} \mid \operatorname{lcm}\left(S_{i}\right) \neq \operatorname{lcm}(S)\right\}=\left\{i \in\{1, \ldots, n\} \mid a_{i} \nmid \operatorname{lcm}\left(S_{i}\right)\right\} .
\end{aligned}
$$

Then we define the type and the cotype of $S$ by:

$$
\operatorname{type}(S)=\left|J^{*}(S)\right| \text { and } \operatorname{cotype}(S)=|J(S)| .
$$

Note that type $(S)$, cotype $(S) \in\{0,1, \ldots, n\}$ and that, if $S^{\prime}$ is the normalization of $S$, then $\operatorname{type}(S)=\operatorname{type}\left(S^{\prime}\right)$ and $\operatorname{cotype}(S)=\operatorname{cotype}\left(S^{\prime}\right)$.

3.10. Proposition. Let $\mathbf{k}$ be a field of characteristic zero and $B$ a $\mathbb{Z}$-graded $\mathbf{k}$-domain. Suppose that there exist homogeneous prime elements $x_{1}, \ldots, x_{n}(n \geq 2)$ of $B$ satisfying the following conditions, where $d_{i}=\operatorname{deg} x_{i}$ for all $i$ :

- $\left\langle d_{1}, \ldots, d_{n}\right\rangle=\mathbb{Z}(B)$ and type $\left(d_{1}, \ldots, d_{n}\right)=0$

- for any choice of distinct $i, j$, the elements $x_{i}, x_{j}$ are not associates in $B$.

Then the following hold.

(a) $\mathbb{Z}(\operatorname{ker} D)=\mathbb{Z}(B)$ for all $D \in \operatorname{HLND}(B)$.

(b) If $B$ is $\mathbf{k}$-affind and not rigid then $\operatorname{HFrac}(B)$ is ruled over $\mathbf{k}$.

Proof. The assumption $\left\langle d_{1}, \ldots, d_{n}\right\rangle=\mathbb{Z}(B)$ and type $\left(d_{1}, \ldots, d_{n}\right)=0$ is equivalent to:

for each subset $I \subset\{1, \ldots, n\}$ of cardinality $n-1,\left\{\operatorname{deg}\left(x_{i}\right) \mid i \in I\right\}$ generates $\mathbb{Z}(B)$.

So assertion (a) is the special case $G=\mathbb{Z}$ of [5. Corollary 4.2]. To prove (b), suppose that $B$ is k-affine and not rigid; then 3.8 implies that $\operatorname{HLND}(B) \backslash\{0\} \neq \varnothing$. Pick $D \in \operatorname{HLND}(B) \backslash\{0\}$, then $\mathbb{Z}(\operatorname{ker} D)=\mathbb{Z}(B)$ by part (a), so Theorem [3.6(c) implies that $\operatorname{HFrac}(B)$ is ruled over $\mathbf{k}$.

\section{Rigidity of Pham-Brieskorn Rings}

Let $\mathbf{k}$ be a field of characteristic zero.

4.1. Definition. Given $n \geq 3$ and $S=\left(a_{1}, \ldots, a_{n}\right) \in(\mathbb{N} \backslash\{0\})^{n}$, we use the notation

$$
B_{S}=B_{a_{1}, \ldots, a_{n}}=\mathbf{k}\left[X_{1}, \ldots, X_{n}\right] /\left\langle X_{1}^{a_{1}}+\cdots+X_{n}^{a_{n}}\right\rangle .
$$

The k-domain $B_{a_{1}, \ldots, a_{n}}$ is called a Pham-Brieskorn ring. We use the capital letters $X_{i}$ to represent the variables in the polynomial ring, and define $x_{i}=\pi\left(X_{i}\right) \in B_{S}$ where $\pi: \mathbf{k}\left[X_{1}, \ldots, X_{n}\right] \rightarrow B_{S}$ is the canonical quotient map. Thus $B_{S}=\mathbf{k}\left[x_{1}, \ldots, x_{n}\right]$. Let $\left(d_{1}, \ldots, d_{n}\right)=\left(\frac{L}{a_{1}}, \ldots, \frac{L}{a_{n}}\right)$, where $L=\operatorname{lcm}(S)$; then there is a unique $\mathbb{N}$-grading of $B_{S}$ with the property that $x_{i}$ is homogeneous of degree $d_{i}$ for all $i=1, \ldots, n$. We call it the standard $\mathbb{N}$-grading of $B_{S}$.

4.2. Lemma. Given $n \geq 2$ and $S=\left(a_{1}, \ldots, a_{n}\right) \in(\mathbb{N} \backslash\{0\})^{n}$, define $\bar{S}=\left(\frac{L}{a_{1}}, \ldots, \frac{L}{a_{n}}\right)$ where $L=\operatorname{lcm}(S)$. Then the following hold.

(a) $\bar{S}$ is normal and $\overline{(\bar{S})}$ is the normalization of $S$.

(b) $J^{*}(\bar{S})=J(S)$ and $J^{*}(S)=J(\bar{S})$.

(c) type $(\bar{S})=\operatorname{cotype}(S)$ and type $(S)=\operatorname{cotype}(\bar{S})$.

The proof of Lemma 4.2 is left to the reader. We deduce the following useful triviality:

\footnotetext{
${ }^{2}$ We mean that $B$ is finitely generated as a k-algebra.
} 
4.3. Lemma. Let $n \geq 4$ and $S=\left(a_{1}, \ldots, a_{n}\right) \in(\mathbb{N} \backslash\{0\})^{n}$, and consider $B_{S}, x_{1}, \ldots, x_{n}$ and $\left(d_{1}, \ldots, d_{n}\right)$ as in Definition 4.1 .

(a) $\operatorname{gcd}\left(d_{1}, \ldots, d_{n}\right)=1$ and type $\left(d_{1}, \ldots, d_{n}\right)=\operatorname{cotype}\left(a_{1}, \ldots, a_{n}\right)$

(b) For each $i \in\{1, \ldots, n\}$, we have

$$
\operatorname{gcd}\left(d_{1}, \ldots \widehat{d}_{i} \ldots, d_{n}\right) \neq 1 \Longleftrightarrow \operatorname{lcm}\left(a_{1}, \ldots \widehat{a}_{i} \ldots, a_{n}\right) \neq \operatorname{lcm}(S) \Longleftrightarrow i \in J(S) .
$$

(c) $x_{1}, \ldots, x_{n}$ are homogeneous prime elements of $B_{S}$ and are pairwise non-associates.

Proof. Since $\left(d_{1}, \ldots, d_{n}\right)=\bar{S}$, assertions (a) and (b) follow from Lemma 4.2, For each $i$, define $S_{i}$ as in Definition [3.9, then $B_{S} /\left\langle x_{i}\right\rangle \cong B_{S_{i}}$, which is a domain because $n \geq 4$. So $x_{1}, \ldots, x_{n}$ are homogeneous prime elements of $B_{S}$, and it is clear that they are pairwise non-associates.

Recall that a $\mathbf{k}$-variety $X$ is said to be ruled if the function field of $X$ is ruled over $\mathbf{k}$, in the sense of Definition 3.5 .

4.4. Theorem. Let $n \geq 4$, and let $S \in(\mathbb{N} \backslash\{0\})^{n}$ be such that cotype $(S)=0$.

(a) We have $\mathbb{Z}(\operatorname{ker} D)=\mathbb{Z}$ for all $D \in \operatorname{HLND}\left(B_{S}\right)$.

(b) If $B_{S}$ is not rigid, then the projective $\mathbf{k}$-variety Proj $B_{S}$ is ruled over $\mathbf{k}$.

Proof. By Example 3.4, the function field of $\operatorname{Proj} B_{S}$ is $\operatorname{HFrac}\left(B_{S}\right)$. Consider $x_{1}, \ldots, x_{n}$ and $\left(d_{1}, \ldots, d_{n}\right)$ as in Definition 4.1. By Lemma 4.3, $x_{1}, \ldots, x_{n}$ satisfy the hypothesis of Proposition 3.10 (in particular type $\left.\left(d_{1}, \ldots, d_{n}\right)=\operatorname{cotype}(S)=0\right)$. So Proposition 3.10 implies that (a) and (b) hold.

4.5. Remark. If $a \geq n \geq 4$ then the Fermat variety $F_{a, n}=\operatorname{Proj}\left(\mathbf{k}\left[X_{1}, \ldots, X_{n}\right] /\left\langle X_{1}^{a}+\cdots+X_{n}^{a}\right\rangle\right)$ is not uniruled, hence not ruled. (Over a field of characteristic zero, a smooth hypersurface of degree $d$ in $\mathbb{P}^{N}$ is uniruled if and only if $d \leq N$.)

4.6. Corollary. If $S=(\underbrace{a, \ldots, a}_{n})$ satisfies $a \geq n \geq 4$, then $B_{S}$ is rigid.

Proof. Note that cotype $(S)=0$. If $B_{S}$ is not rigid then (by Theorem 4.4) Proj $B_{S}=F_{a, n}$ is ruled, which is not the case by Remark 4.5 .

We shall now develop a different approach for proving that $B_{S}$ is rigid in certain cases. We need the following result, which is part (b) of Corollary 3.3 of [10]. See 2.3 for the definition of $|u|_{A}$.

4.7. Corollary. Suppose $R$ is a $\mathbb{Z}$-graded affine $\mathbf{k}$-domain, $f \in R$ is homogeneous, and $n \geq 2$ is an integer not dividing $\operatorname{deg} f$. Set $g=\operatorname{gcd}(n, \operatorname{deg} f)$, define the rings

$$
A=R[u] /\left\langle f+u^{g}\right\rangle \text { and } B=R[z] /\left\langle f+z^{n}\right\rangle
$$

and assume that $B$ is a domain. Then $A$ is a domain and

$$
B \text { is rigid if and only if }|u|_{A} \geq 2 \text {. }
$$

4.8. Definition. Let $n \geq 3$.

(a) Given $S=\left(a_{1}, \ldots, a_{n}\right) \in(\mathbb{N} \backslash\{0\})^{n}$ and $i \in\{1, \ldots, n\}$, define $g_{i}(S)=\operatorname{gcd}\left(a_{i}, \operatorname{lcm}\left(S_{i}\right)\right)$ (recalling the definition of $S_{i}$ from Definition 3.9). 
(b) Let $S=\left(a_{1}, \ldots, a_{n}\right)$ and $S^{\prime}=\left(a_{1}^{\prime}, \ldots, a_{n}^{\prime}\right)$ be elements of $(\mathbb{N} \backslash\{0\})^{n}$ and let $i \in\{1, \ldots, n\}$. We write $S \leq{ }^{i} S^{\prime}$ if and only if

$$
S_{i}=S_{i}^{\prime} \quad \text { and } \quad g_{i}\left(S^{\prime}\right)\left|a_{i}\right| a_{i}^{\prime} .
$$

We write $S<^{i} S^{\prime}$ if and only if $S \leq^{i} S^{\prime}$ and $S \neq S^{\prime}$.

Observe that $\leq^{i}$ is a partial order on $(\mathbb{N} \backslash\{0\})^{n}$ (transitivity follows from the fact that if $S \leq^{i} S^{\prime}$ then $\left.g_{i}(S)=g_{i}\left(S^{\prime}\right)\right)$.

4.9. Proposition. Let $n \geq 3$.

(a) Suppose that $S^{*}, S \in(\mathbb{N} \backslash\{0\})^{n}$ and $i \in\{1, \ldots, n\}$ satisfy $S^{*} \leq{ }^{i} S$. If $B_{S^{*}}$ is rigid then so is $B_{S}$.

(b) Let $S, S^{\prime} \in(\mathbb{N} \backslash\{0\})^{n}$, and suppose that there exist $i \in\{1, \ldots, n\}$ and $S^{*} \in(\mathbb{N} \backslash\{0\})^{n}$ such that $S^{*}<^{i} S$ and $S^{*}<^{i} S^{\prime}$. Then $B_{S}$ is rigid if and only if $B_{S^{\prime}}$ is rigid.

Proof. (b) We may assume that $i=n$. Consider $S=\left(a_{1}, \ldots, a_{n}\right), S^{\prime}=\left(a_{1}^{\prime}, \ldots, a_{n}^{\prime}\right)$ and $S^{*}=$ $\left(a_{1}^{*}, \ldots, a_{n}^{*}\right)$ satisfying $S^{*}<^{n} S$ and $S^{*}<^{n} S^{\prime}$. The hypothesis implies that

$$
S_{n}=S_{n}^{*}=S_{n}^{\prime}, \quad g\left|a_{n}^{*}\right| a_{n} \text { and } g\left|a_{n}^{*}\right| a_{n}^{\prime},
$$

where $g=g_{n}(S)=g_{n}\left(S^{*}\right)=g_{n}\left(S^{\prime}\right)$. Let $L=\operatorname{lcm}\left(a_{1}, \ldots, a_{n-1}\right)$ and define an $\mathbb{N}$-grading on $R=\mathbf{k}\left[X_{1}, \ldots, X_{n-1}\right]$ by declaring that (for each $i=1, \ldots, n-1$ ) $X_{i}$ is homogeneous of degree $\frac{a_{n}^{*}}{g} \frac{L}{a_{i}}$. Then $f=X_{1}^{a_{1}}+\cdots+X_{n-1}^{a_{n-1}}$ is homogeneous of degree $\frac{a_{n}^{*} L}{g}$. Consider the rings

$$
A=R[u] /\left\langle f+u^{a_{n}^{*}}\right\rangle, \quad B=R[z] /\left\langle f+z^{a_{n}}\right\rangle, \quad B^{\prime}=R[w] /\left\langle f+w^{a_{n}^{\prime}}\right\rangle .
$$

Observe that $A \cong B_{S^{*}}, B \cong B_{S}$ and $B^{\prime} \cong B_{S^{\prime}}$. The reader may verify that $a_{n} \nmid \operatorname{deg} f, a_{n}^{\prime} \nmid \operatorname{deg} f$ and $\operatorname{gcd}\left(a_{n}, \operatorname{deg} f\right)=a_{n}^{*}=\operatorname{gcd}\left(a_{n}^{\prime}, \operatorname{deg} f\right)$; thus each one of the pairs $(A, B),\left(A, B^{\prime}\right)$ satisfies the hypothesis of Corollary 4.7. By that result, $B$ is rigid $\Leftrightarrow|u|_{A} \geq 2 \Leftrightarrow B^{\prime}$ is rigid, so (b) is proved. It also follows that (a) is true, because if $A$ is rigid then $|u|_{A}=\infty \geq 2$, so $B$ is rigid.

\subsection{Corollary.}

(a) If $k_{1}, k_{2}, k_{3}, k_{4}$ are pairwise relatively prime positive integers and $a \geq 3$ then $B_{a k_{1}, a k_{2}, a k_{3}, a k_{4}}$ is rigid.

(b) If $k_{1}, \ldots, k_{n}$ are pairwise relatively prime positive integers and $a \geq n \geq 4$ then $B_{a k_{1}, \ldots, a k_{n}}$ is rigid.

Proof. Define $S^{0}=(a, a, a, a)$ to prove $(\mathrm{a})$, and $S^{0}=(\overbrace{a, \ldots, a}^{n})$ to prove (b). Note that $B_{S^{0}}$ is rigid (by Theorem [1.3(c) if $S^{0}=(3,3,3,3)$, by Corollary 4.6 in the other cases). Define $S^{1}=\left(a k_{1}, a, a, \ldots\right), S^{2}=\left(a k_{1}, a k_{2}, a, \ldots\right), \ldots, S^{n-1}=\left(a k_{1}, \ldots, a k_{n-1}, a\right), S^{n}=\left(a k_{1}, \ldots, a k_{n}\right)$; then $S^{0} \leq^{1} S^{1} \leq^{2} S^{2} \leq^{3} \cdots \leq^{n-1} S^{n-1} \leq^{n} S^{n}$. Since $B_{S^{0}}$ is rigid, so is $B_{S^{n}}$ by Proposition 4.9.

4.11. Lemma. Given $n \geq 3$ and $S=\left(a_{1}, \ldots, a_{n}\right) \in(\mathbb{N} \backslash\{0\})^{n}$,

$$
J(S)=\left\{j \mid S \text { is not minimal with respect to }<^{j}\right\} .
$$

Proof. If $j \in J(S)$ then $a_{j} \nmid \operatorname{lcm}\left(S_{j}\right)$, so $g_{j}(S) \neq a_{j}$ and hence

$$
\left(a_{1}, \ldots, a_{j-1}, g_{j}(S), a_{j+1}, \ldots, a_{n}\right)<^{j} S .
$$

Conversely, if $\left(a_{1}^{\prime}, \ldots, a_{n}^{\prime}\right)<^{j} S$ then $g_{j}(S)\left|a_{j}^{\prime}\right| a_{j}$ and $a_{j}^{\prime} \neq a_{j}$, so $j \in J(S)$. 
4.12. Proposition. Let $n \geq 3, S=\left(a_{1}, \ldots, a_{n}\right) \in(\mathbb{N} \backslash\{0\})^{n}$ and

$$
I(S)=\{1, \ldots, n\} \backslash J(S)=\left\{i \mid a_{i} \text { divides } \operatorname{lcm}\left(S_{i}\right)\right\} .
$$

If $\sum_{i \in I(S)} \frac{1}{a_{i}}<\frac{1}{n-2}$ then $B_{S}$ is rigid.

Proof. Let us write $I=I(S)$ and $J=J(S)$. If $J=\varnothing$ then $\sum_{i=1}^{n} \frac{1}{a_{i}}<\frac{1}{n-2}$ so we are done by Theorem 1.2. Assume that $J \neq \varnothing$ and let $j_{1}<\cdots<j_{k}$ be the elements of $J$. Choose distinct primes $p_{j_{1}}, \ldots, p_{j_{k}}$ such that $\operatorname{gcd}\left(\prod_{j \in J} p_{j}, \prod_{i=1}^{n} a_{i}\right)=1$. Choose positive integers $e_{j_{1}}, \ldots, e_{j_{k}}$ such that $\sum_{i \in I} \frac{1}{a_{i}}+\sum_{j \in J} \frac{1}{a_{j} p_{j}^{e_{j}}}<\frac{1}{n-2}$. We inductively define a sequence $S^{0}, S^{1}, \ldots, S^{k}$ of elements of $(\mathbb{N} \backslash\{0\})^{n}$ by setting $S^{0}=S$ and, for each $\nu=1, \ldots, k$,

$$
S^{\nu}=\text { componentwise product }\left(1, \ldots, 1, p_{j_{\nu}}^{e_{j_{\nu}}}, 1, \ldots, 1\right) \cdot S^{\nu-1}
$$

where the $p_{j_{\nu}}^{e_{j_{\nu}}}$ is in the $j_{\nu}$-th position. The reader can check that $J\left(S^{\nu}\right)=J(S)=J$ for all $\nu$ and that $S^{0} \leq^{j_{1}} S^{1} \leq^{j_{2}} S^{2} \leq^{j_{3}} \ldots \leq^{j_{k}} S^{k}$.

Let $\nu \in\{1, \ldots, k\}$; since $j_{\nu} \in J=J\left(S^{\nu-1}\right)$, Lemma 4.11 implies that $S^{\nu-1}$ is not minimal with respect to $\leq^{j_{\nu}}$, i.e., there exists $S_{*}^{\nu-1} \in(\mathbb{N} \backslash\{0\})^{n}$ satisfying $S_{*}^{\nu-1}<^{j_{\nu}} S^{\nu-1}<^{j_{\nu}} S^{\nu}$; then Proposition 4.9(b) implies that $B_{S^{\nu-1}}$ is rigid if and only if $B_{S^{\nu}}$ is rigid. As this holds for all $\nu \in\{1, \ldots, k\}, B_{S}=B_{S^{0}}$ is rigid if and only if $B_{S^{k}}$ is rigid. Now $B_{S^{k}}$ is indeed rigid by Theorem 1.2, because if we write $S^{k}=\left(a_{1}^{\prime}, \ldots, a_{n}^{\prime}\right)$ then $\sum_{i=1}^{n} \frac{1}{a_{i}^{\prime}}=\sum_{i \in I} \frac{1}{a_{i}}+\sum_{j \in J} \frac{1}{a_{j} e_{j}^{e_{j}}}<\frac{1}{n-2}$. So $B_{S}$ is rigid.

Proposition 4.12 shows that many Pham-Brieskorn varieties that are not shown to be rigid by Theorems 1.1 1.3 are indeed rigid. In the case $n=4$, the following is an immediate consequence of Proposition 4.12:

4.13. Corollary. If $a, b, c, d \in \mathbb{N} \backslash\{0\}$ satisfy $a \nmid \operatorname{lcm}(b, c, d)$ and $\frac{1}{b}+\frac{1}{c}+\frac{1}{d}<\frac{1}{2}$ then $B_{a, b, c, d}$ is rigid.

We need the following:

4.14. Corollary. Let $n \geq 4$ and $S \in(\mathbb{N} \backslash\{0\})^{n}$. Consider $B_{S}=\mathbf{k}\left[x_{1}, \ldots, x_{n}\right]$ with $x_{1}, \ldots, x_{n}$ as in Definition 4.1. Then for every $D \in \operatorname{HLND}\left(B_{S}\right)$ the following conditions hold.

(a) If $j \in J(S)$ then $D^{2}\left(x_{j}\right)=0$.

(b) If $j_{1}, j_{2}$ are distinct elements of $J(S)$ then $D\left(x_{j_{1}}\right)=0$ or $D\left(x_{j_{2}}\right)=0$.

Proof. In view of Lemma 4.3(b), this is a special case of Corollary 6.3 of [5].

4.15. Proposition. Let $n \geq 4$ and $S \in(\mathbb{N} \backslash\{0\})^{n}$. Assume that $J(S) \neq \varnothing$ and that

$$
B_{S_{J}} \text { is rigid for every subset } J \text { of } J(S) \text { satisfying }|J|=\min (|J(S)|-1, n-3) \text {. }
$$

Then $B_{S}$ is rigid.

Proof. If $|J(S)|=1$ then $\min (|J(S)|-1, n-3)=0$, so $(*)$ reads " $B_{S}$ is rigid," so the Proposition is trivially true in this case. From now-on, we assume that $|J(S)|>1$. By contradiction, assume that $B_{S}$ is not rigid. Then $\operatorname{HLND}\left(B_{S}\right) \neq\{0\}$ by 3.8, choose an irreducible $D \in \operatorname{HLND}\left(B_{S}\right) \backslash\{0\}$ (this is possible by 2.2( (c) ). By Corollary 4.14 , we have $D\left(x_{j_{1}}\right)=0$ or $D\left(x_{j_{2}}\right)=0$ for every choice of distinct $j_{1}, j_{2} \in J(S)$. So there exists a subset $J^{\prime}$ of $J(S)$ such that $\left|J^{\prime}\right|=|J(S)|-1$ and $D\left(x_{j}\right)=0$ for all $j \in J^{\prime}$; hence there exists a $J \subseteq J^{\prime} \subset J(S)$ such that $|J|=\min (|J(S)|-1, n-3)$ and $D\left(x_{j}\right)=0$ for all $j \in J$. We might as well assume that $J=\{m+1, m+2, \ldots, n\}$ for some $m \geq 3$. For each $i$ such that $m \leq i \leq n$ we define $P(i)$ to be the statement 
there exists an irreducible $D_{i} \in \operatorname{HLND}\left(B_{a_{1}, \ldots, a_{i}}\right) \backslash\{0\}$

satisfying $D_{i}\left(x_{j}\right)=0$ for all $j \in\{1, \ldots, i\} \cap J$

where $x_{j}$ denotes the image of $X_{j}$ in $B_{a_{1}, \ldots, a_{i}}$. Then $P(n)$ is true, with $D_{n}=D$. Assume that $i$ is such that $m<i \leq n$ and such that $P(i)$ is true. Note that $i \in J$, so $D_{i}\left(x_{i}\right)=0$, so $D_{i}$ induces a homogeneous locally nilpotent derivation $\bar{D}_{i}$ of the $\operatorname{ring} B_{a_{1}, \ldots, a_{i}} /\left\langle x_{i}\right\rangle \cong B_{a_{1}, \ldots, a_{i-1}}$, and $\bar{D}_{i} \neq 0$ because $D_{i}$ is irreducible. Moreover, $\bar{D}_{i}\left(\bar{x}_{j}\right)=0$ for all $j \in\{1, \ldots, i-1\} \cap J$. By 2.2((c)), there exists an irreducible $D_{i-1} \in \operatorname{HLND}\left(B_{a_{1}, \ldots, a_{i-1}}\right) \backslash\{0\}$ such that $\operatorname{ker} D_{i-1}=\operatorname{ker} \bar{D}_{i}$, so $P(i-1)$ is true. By descending induction, $P(n), P(n-1), \ldots, P(m)$ are all true. Since $P(m)$ is true and $S_{J}=\left(a_{1}, \ldots, a_{m}\right)$, we have $D_{m} \in \operatorname{HLND}\left(B_{S_{J}}\right) \backslash\{0\}$, so $B_{S_{J}}$ is not rigid, contradicting $(*)$.

We generalize Theorem 1.3(e):

4.16. Corollary. Let $n \geq 4$ and $S \in T_{n}$. If $\operatorname{cotype}(S) \geq n-2$, then $B_{S}$ is rigid.

Proof. We have $|J(S)|=\operatorname{cotype}(S) \geq n-2$, so $\min (|J(S)|-1, n-3)=n-3$. Let $J$ be any subset of $J(S)$ satisfying $|J|=\min (|J(S)|-1, n-3)=n-3$; since $S \in T_{n}$, we have $S_{J} \in T_{3}$, so $B_{S_{J}}$ is rigid by Theorem 1.1. Then Proposition 4.15 implies that $B_{S}$ is rigid.

4.17. Example. Proposition 4.15 can settle cases not covered by Corollary 4.16 or by any of the previous results. For instance:

If $a_{1}, \ldots, a_{m} \in(\mathbb{N} \backslash\{0\})^{m}(m \geq 1)$ satisfy $a_{i} \nmid \operatorname{lcm}\left(3, a_{1}, \ldots \widehat{a_{i}} \ldots, a_{m}\right)$ for all $i \in\{1, \ldots, m\}$, then $B_{a_{1}, \ldots, a_{m}, 3,3,3}$ is rigid.

Indeed, let $S=\left(a_{1}, \ldots, a_{m}, 3,3,3\right)$, then $J(S)=\{1, \ldots, m\}$ and (by Theorem $\left.1.3(\mathrm{c})\right) B_{a_{i}, 3,3,3}$ is rigid for each $i \in J(S)$, so $B_{S}$ is rigid by Proposition 4.15, For instance, $B_{2,5,7,3,3,3}$ is rigid.

4.18. Notation. Given $S=\left(a_{1}, \ldots, a_{n}\right) \in(\mathbb{N} \backslash\{0\})^{n}(n \geq 2)$ and a subset $M$ of $\{1, \ldots, n\}$, define a positive integer $\Delta_{M}(S)$ by $\Delta_{\varnothing}(S)=1$ and

$$
\Delta_{M}(S)=\frac{\operatorname{lcm}(S)}{\operatorname{gcd}\left\{\operatorname{lcm}\left(S_{j}\right) \mid j \in M\right\}} \quad \text { if } M \neq \varnothing .
$$

Observe that

$$
\Delta_{M}(S)=\Delta_{J(S) \cap M}(S) \text { and } \Delta_{M}(S)=1 \Leftrightarrow M \cap J(S)=\varnothing,
$$

because $\operatorname{lcm}\left(S_{j}\right)=\operatorname{lcm}(S)$ for each $j \in\{1, \ldots, n\} \backslash J(S)$, and $\operatorname{lcm}\left(S_{j}\right)$ is a proper divisor of $\operatorname{lcm}(S)$ for each $j \in J(S)$.

4.19. Proposition. Let $n \geq 4, S \in(\mathbb{N} \backslash\{0\})^{n}$ and $M=\left\{j \in J(S) \mid B_{S_{j}}\right.$ is rigid $\}$.

(a) $\mathbb{Z}(\operatorname{ker} D) \subseteq \Delta_{M}(S) \cdot \mathbb{Z}$ for all $D \in \operatorname{HLND}\left(B_{S}\right) \backslash\{0\}$.

(b) $\Delta_{M}(S)=1 \Longleftrightarrow M=\varnothing$

Proof. We use the following notation: $S=\left(a_{1}, \ldots, a_{n}\right), \bar{S}=\left(d_{1}, \ldots d_{n}\right), x_{1}, \ldots, x_{n}$ as in Definition 4.1, $L=\operatorname{lcm}(S)$ and, for each $i \in\{1, \ldots, n\}, L_{i}=\operatorname{lcm}\left(S_{i}\right)$ and $\delta_{i}=\operatorname{gcd}\left(d_{1}, \ldots \widehat{d}_{i} \ldots, d_{n}\right)$. Then $\delta_{i}=\operatorname{gcd}\left(\frac{L}{a_{1}}, \ldots \frac{\widehat{L}}{a_{i}} \ldots, \frac{L}{a_{n}}\right)=\frac{L}{L_{i}} \operatorname{gcd}\left(\frac{L_{i}}{a_{1}}, \ldots \frac{\widehat{L_{i}}}{a_{i}} \ldots, \frac{L_{i}}{a_{n}}\right)=\frac{L}{L_{i}}$ for each $i \in\{1, \ldots, n\}$, so $\operatorname{lcm}\left(\delta_{j}: j \in M\right)=\operatorname{lcm}\left(\frac{L}{L_{j}}: j \in M\right)=\frac{L}{\operatorname{gcd}\left(L_{j}: j \in M\right)}=\Delta_{M}(S)$ and consequently $\bigcap_{j \in M} \delta_{j} \mathbb{Z}=$ $\Delta_{M}(S) \cdot \mathbb{Z}$. So, to prove (a), it suffices to show that

$$
\text { for each } D \in \operatorname{HLND}\left(B_{S}\right) \backslash\{0\} \text { and each } j \in M, \quad \mathbb{Z}(\operatorname{ker} D) \subseteq \delta_{j} \mathbb{Z} \text {. }
$$

We prove this by contradiction: suppose that $D \in \operatorname{HLND}\left(B_{S}\right) \backslash\{0\}$ and $j \in M$ satisfy $\mathbb{Z}($ ker $D) \nsubseteq$ $\delta_{j} \mathbb{Z}$. By $2.2(\mathbb{C})$, there exists an irreducible $D^{\prime} \in \operatorname{HLND}\left(B_{S}\right) \backslash\{0\}$ with $\operatorname{ker}\left(D^{\prime}\right)=\operatorname{ker}(D)$, so we 
may choose $D$ irreducible. Then there exists a homogeneous element $h \in \operatorname{ker}(D) \backslash\{0\}$ such that $\operatorname{deg}(h) \notin \delta_{j} \mathbb{Z}$. We can write $h=\sum_{\left(i_{1}, \ldots, i_{n}\right) \in E} \lambda_{i_{1}, \ldots, i_{n}} x_{1}^{i_{1}} \cdots x_{n}^{i_{n}}$ for some finite set $E \subset \mathbb{N}^{n}$, where for each $\left(i_{1}, \ldots, i_{n}\right) \in E$ we have $\lambda_{i_{1}, \ldots, i_{n}} \in \mathbf{k}^{*}$ and $\operatorname{deg}\left(x_{1}^{i_{1}} \cdots x_{n}^{i_{n}}\right)=\operatorname{deg}(h) \notin \delta_{j} \mathbb{Z}$. So for each $\left(i_{1}, \ldots, i_{n}\right) \in E$ we have $\operatorname{deg}\left(x_{j}^{i_{j}}\right) \notin \delta_{j} \mathbb{Z}$ and in particular $i_{j}>0$. This shows that $x_{j} \mid h$ in $B_{S}$. Since $\operatorname{ker}(D)$ is factorially closed in $B_{S}$, we get $x_{j} \in \operatorname{ker} D$. Then $D$ induces a locally nilpotent derivation $\bar{D}$ of the ring $B_{S} /\left\langle x_{j}\right\rangle \cong B_{S_{j}}$, and $\bar{D} \neq 0$ because $D\left(B_{S}\right) \nsubseteq\left\langle x_{j}\right\rangle$ (since $D$ is irreducible). So $B_{S_{j}}$ is not rigid, which contradicts $j \in M$. This proves (1), so assertion (a) is proved. Part (b) follows from the observation (made in 4.18) that, for any subset $M$ of $\{1, \ldots, n\}$, we have $\Delta_{M}(S)=1$ if and only if $M \cap J(S)=\varnothing$.

It immediately follows:

4.20. Corollary. Let $n \geq 4$ and $S \in(\mathbb{N} \backslash\{0\})^{n}$. If some $D \in \operatorname{HLND}\left(B_{S}\right) \backslash\{0\}$ satisfies $\mathbb{Z}(\operatorname{ker} D)=$ $\mathbb{Z}$ then $B_{S_{j}}$ is non-rigid for every $j \in J(S)$.

Here is another consequence of Proposition 4.19, valid for $n=4$ :

4.21. Corollary. Let $S \in T_{4}$ be such that $\operatorname{cotype}(S)>0$ and define $\delta=\Delta_{J(S)}(S) \in \mathbb{Z}$. Then

$$
\delta>1 \quad \text { and } \quad \mathbb{Z}(\operatorname{ker} D) \subseteq \delta \mathbb{Z} \text { for all } D \in \operatorname{HLND}\left(B_{S}\right) \backslash\{0\} .
$$

Proof. We have $J(S) \neq \varnothing$ because $\operatorname{cotype}(S)>0$. If $j \in J(S)$ then $S_{j} \in T_{3}$, so $B_{S_{j}}$ is rigid by Theorem 1.1. So the set $M=\left\{j \in J(S) \mid B_{S_{j}}\right.$ is rigid $\}$ of Proposition 4.19 is equal to $J(S)$ (which is not empty). The desired conclusion follows from Proposition 4.19.

\section{A Remark about $\operatorname{Proj}\left(B_{S}\right)$}

As in the preceding section, we assume that $\mathbf{k}$ is a field of characteristic zero.

5.1. Proposition (Exercise 9.5 in [7]). Let $R=R_{0} \oplus R_{1} \oplus R_{2} \oplus \ldots$ be an $\mathbb{N}$-graded Noetherian ring, let $d>0$ and let $R^{(d)}=R_{0} \oplus R_{d} \oplus R_{2 d} \oplus \ldots$ Then $\operatorname{Proj} R \cong \operatorname{Proj} R^{(d)}$.

5.2. Proposition. Let $S=\left(a_{1}, \ldots, a_{n}\right), S^{\prime}=\left(a_{1}^{\prime}, \ldots, a_{n}^{\prime}\right) \in(\mathbb{N} \backslash\{0\})^{n}(n \geq 3)$ and assume that $i \in\{1, \ldots, n\}$ is such that $S \leq^{i} S^{\prime}$. Then $B_{S} \cong B_{S^{\prime}}^{(k)}$, where we define $k=a_{i}^{\prime} / a_{i} \in \mathbb{N} \backslash\{0\}$. Consequently, Proj $B_{S} \cong \operatorname{Proj} B_{S^{\prime}}$.

Proof. We may assume that $i=1$. Define $\left(d_{1}, \ldots, d_{n}\right)=\bar{S}$ and $\left(d_{1}^{\prime}, \ldots, d_{n}^{\prime}\right)=\overline{S^{\prime}}$. Let us prove:

$$
\left(d_{1}^{\prime}, \ldots, d_{n}^{\prime}\right)=\left(d_{1}, k d_{2}, \ldots, k d_{n}\right) \text { and } \operatorname{gcd}\left(d_{1}, k\right)=1 \text {. }
$$

Let $L=\operatorname{lcm}(S), L^{\prime}=\operatorname{lcm}\left(S^{\prime}\right)$, and $L_{1}=\operatorname{lcm}\left(S_{1}\right)=\operatorname{lcm}\left(S_{1}^{\prime}\right)$. Let $g_{1}=g_{1}\left(S^{\prime}\right)=g_{1}(S)$, i.e., $g_{1}=\operatorname{gcd}\left(a_{1}^{\prime}, L_{1}\right)=\operatorname{gcd}\left(a_{1}, L_{1}\right)$. We have $L=\operatorname{lcm}\left(a_{1}, L_{1}\right)=a_{1} L_{1} / g_{1}$ and $L^{\prime}=\operatorname{lcm}\left(a_{1}^{\prime}, L_{1}\right)=$ $a_{1}^{\prime} L_{1} / g_{1}$, so for each $j \in\{1, \ldots, n\}$, we have $d_{j}=L / a_{j}=\frac{a_{1} L_{1}}{a_{j} g_{1}}$ and $d_{j}^{\prime}=L^{\prime} / a_{j}^{\prime}=\frac{a_{1}^{\prime} L_{1}}{a_{j}^{\prime} g_{1}}$. This gives $d_{1}^{\prime}=L_{1} / g_{1}=d_{1}$ and, for $j \neq 1, d_{j}^{\prime}=\frac{a_{1}^{\prime} L_{1}}{a_{j}^{\prime} g_{1}}=\frac{k a_{1} L_{1}}{a_{j} g_{1}}=k d_{j}$; this proves the first part of (2). Since $\operatorname{gcd}\left(d_{1}, k\right)$ is a divisor of $\operatorname{gcd}\left(d_{1}, k d_{2}, \ldots, k d_{n}\right)=\operatorname{gcd}\left(d_{1}^{\prime}, \ldots, d_{n}^{\prime}\right)=1$, (2) is proved. Now let $\Phi: \mathbf{k}\left[X_{1}, \ldots, X_{n}\right] \rightarrow \mathbf{k}\left[Y_{1}, \ldots, Y_{n}\right]$ be the $\mathbf{k}$-homomorphism that sends $X_{1}$ to $Y_{1}^{k}$ and $X_{j}$ to $Y_{j}$ for $j>1$. Noting that $Y_{1}^{a_{1}^{\prime}}+\cdots+Y_{n}^{a_{n}^{\prime}}=Y_{1}^{k a_{1}}+Y_{2}^{a_{2}}+\cdots+Y_{n}^{a_{n}}$, we see that $\Phi^{-1}\left(\left\langle Y_{1}^{a_{1}^{\prime}}+\cdots+Y_{n}^{a_{n}^{\prime}}\right\rangle\right)=$ $\left\langle X_{1}^{a_{1}}+\cdots+X_{n}^{a_{n}}\right\rangle$, so $\Phi$ induces an injective homomorphism

$$
\varphi: B_{S}=\mathbf{k}\left[X_{1}, \ldots, X_{n}\right] /\left\langle X_{1}^{a_{1}}+\cdots+X_{n}^{a_{n}}\right\rangle \rightarrow B_{S^{\prime}}=\mathbf{k}\left[Y_{1}, \ldots, Y_{n}\right] /\left\langle Y_{1}^{a_{1}^{\prime}}+\cdots+Y_{n}^{a_{n}^{\prime}}\right\rangle .
$$


Then $B_{S} \cong \varphi\left(B_{S}\right)=\mathbf{k}\left[y_{1}^{k}, y_{2}, \ldots, y_{n}\right]$. Since $\operatorname{deg}\left(y_{j}\right)=d_{j}^{\prime}$ for all $j$, (2) implies that $B_{S^{\prime}}^{(k)}=$ $\mathbf{k}\left[y_{1}^{k}, y_{2}, \ldots, y_{n}\right]$ and that $\varphi$ is homogeneous (meaning $\varphi\left(\left(B_{S}\right)_{i}\right) \subseteq\left(B_{S^{\prime}}\right)_{k i}$ for all $i \in \mathbb{N}$ ); so $B_{S} \cong B_{S^{\prime}}^{(k)}$ as graded rings. Then Proj $B_{S} \cong \operatorname{Proj} B_{S^{\prime}}$ follows from Proposition 5.1.

5.3. Remark. It is interesting to observe that the geometry of Proj $B_{S}$ is in general not sufficient to determine whether or not $B_{S}$ is rigid (whereas the geometry of Spec $B_{S}$ is of course sufficient). For example, let $S=(2,3,3,2), S^{\prime}=(2,3,3,4)$ and $S^{\prime \prime}=(10,3,3,4)$. Since $S<4 S^{\prime}<^{1} S^{\prime \prime}$, Proposition 5.2 implies that Proj $B_{S} \cong \operatorname{Proj} B_{S^{\prime}} \cong \operatorname{Proj} B_{S^{\prime \prime}}$. However, $B_{S}=B_{2,3,3,2}$ is not rigid because $(2,3,3,2) \notin T_{4}$, and $B_{S^{\prime \prime}}=B_{10,3,3,4}$ is rigid by Theorem 1.3(e). Interestingly, we don't know whether $B_{S^{\prime}}$ is rigid or not.

\section{Stable Rigidity}

6.1. Lemma. Let $E \subseteq F$ be a field extension, $m \geq 1, t_{1}, \ldots, t_{m}$ independent indeterminates over $F$ and $f, g \in E\left[t_{1}, \ldots, t_{m}\right] \subseteq F\left[t_{1}, \ldots, t_{m}\right]$. Then $f, g$ are relatively prime in $E\left[t_{1}, \ldots, t_{m}\right]$ if and only if they are relatively prime in $F\left[t_{1}, \ldots, t_{m}\right]$.

Verification of the above Lemma is left to the reader. We quote Theorem 3.1 of [6]:

6.2. Theorem. Let $m \geq 1, n \geq 3, g_{1}, \ldots, g_{n} \in \mathbb{C}\left[t_{1}, \ldots, t_{m}\right]=\mathbb{C}^{[m]}$ and $a_{1}, \ldots, a_{n} \in \mathbb{N} \backslash\{0\}$ be such that:

- $g_{1}^{a_{1}}+\cdots+g_{n}^{a_{n}}=0$

- $g_{1}, \ldots, g_{n}$ are not all constant

- given any $1 \leq i_{1}<\cdots<i_{s} \leq n$ such that $g_{i_{1}}^{a_{i_{1}}}+\cdots+g_{i_{s}}^{a_{i_{s}}}=0$, we have $\operatorname{gcd}\left(g_{i_{1}}, \ldots, g_{i_{s}}\right)=1$. Then $\sum_{i=1}^{n} \frac{1}{a_{i}}>\frac{1}{d-1}$ where $d$ is the dimension of the $\mathbb{C}$-vector space spanned by $g_{1}^{a_{1}}, \ldots, g_{n}^{a_{n}}$.

6.3. Corollary. Let $K$ be a field of characteristic zero, $m \geq 1, n \geq 3, g_{1}, \ldots, g_{n} \in K\left[t_{1}, \ldots, t_{m}\right]=$ $K^{[m]}$ and $a_{1}, \ldots, a_{n} \in \mathbb{N} \backslash\{0\}$ be such that:

(i) $g_{1}^{a_{1}}+\cdots+g_{n}^{a_{n}}=0$

(ii) $\sum_{i=1}^{n} \frac{1}{a_{i}} \leq \frac{1}{n-2}$

(iii) $g_{1}, \ldots, g_{n}$ are pairwise relatively prime.

Then $g_{1}, \ldots, g_{n} \in K$.

Proof. First consider the case where $K=\mathbb{C}$. Let $d$ be the dimension of the $\mathbb{C}$-vector space spanned by $g_{1}^{a_{1}}, \ldots, g_{n}^{a_{n}}$. Note that $d<n$ by (i); if $d \leq 1$ then the conclusion $\left(g_{1}, \ldots, g_{n} \in K\right)$ immediately follows from (iii), so we may assume that $1<d<n$. Then $\frac{1}{d-1}$ is defined and $\frac{1}{d-1} \geq \frac{1}{n-2}$. So (ii) gives $\sum_{i=1}^{n} \frac{1}{a_{i}} \leq \frac{1}{d-1}$ and Theorem 6.2 implies that $g_{1}, \ldots, g_{n} \in K$. So the case $K=\mathbb{C}$ of Corollary 6.3 is true.

Now let $K$ be arbitrary. Let $K_{0} \subseteq K$ be the extension of $\mathbb{Q}$ generated by the coefficients of $g_{1}, \ldots, g_{n}$. Then $K_{0}$ can be embedded in $\mathbb{C}$; more precisely, if we choose a sufficiently large overfield $L$ of $K$ then we may find a copy of $\mathbb{C}$ in $L$ forming a diagram of fields as in the left part of:
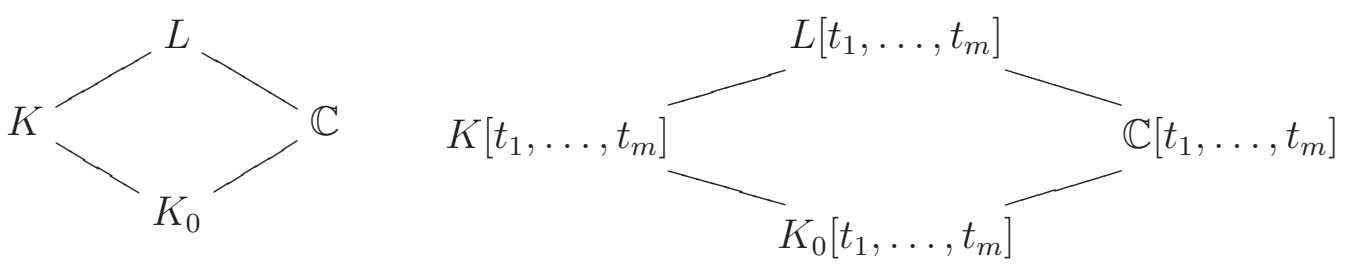
Now $g_{1}, \ldots, g_{n} \in K_{0}\left[t_{1}, \ldots, t_{m}\right] \subseteq \mathbb{C}\left[t_{1}, \ldots, t_{m}\right]$ are pairwise relatively prime in $\mathbb{C}\left[t_{1}, \ldots, t_{m}\right]$ by Lemma 6.1. So $g_{1}, \ldots, g_{n}$ satisfy (i-iii) as elements of $\mathbb{C}\left[t_{1}, \ldots, t_{m}\right]$. By the case $K=\mathbb{C}$ of Corollary 6.3, we get $g_{1}, \ldots, g_{n} \in \mathbb{C}$, so $g_{1}, \ldots, g_{n} \in K$.

We need the notion of relatively prime elements of an arbitrary domain.

6.4. Definition. Let $x, y \in B$ where $B$ is a domain. One says that $x, y$ are relatively prime in $B$ if the following hold:
(i) $x B \cap y B=x y B$
(ii) if $0 \in\{x, y\}$ then $\{x, y\} \cap B^{*} \neq \varnothing$.

Note that this agrees with the usual notion when $B$ is a UFD.

6.5. Remark. Let $x, y$ be relatively prime elements of a domain $B$.

(a) If $S$ is a multiplicative set of $B$ such that $0 \notin S$, then $x, y$ are relatively prime in $S^{-1} B$.

(b) If $A$ is a factorially closed subring of $B$ such that $x, y \in A$, then $x, y$ are relatively prime in $A$.

(c) If $B^{\prime}=B^{[N]}$ for some $N \geq 0$ then $x, y$ are relatively prime in $B^{\prime}$.

6.6. Definition. Let $B$ be a domain of characteristic zero.

(a) $\operatorname{ML}(B)=\bigcap_{D \in \operatorname{LND}(B)} \operatorname{ker}(D)$

(b) The rigid core of $B$ is defined as $\bigcap_{i=0}^{\infty} \operatorname{ML}_{i}(B)$, where one defines $\operatorname{ML}_{0}(B)=B$ and $\mathrm{ML}_{i+1}(B)=\operatorname{ML}\left(\mathrm{ML}_{i} B\right)$ for all $i$.

The next result generalizes Theorem 6.1(a) of [10].

6.7. Theorem. Let $B$ be a domain of characteristic zero, $n \geq 3, x_{1}, \ldots, x_{n} \in B$ and $a_{1}, \ldots, a_{n} \in$ $\mathbb{N} \backslash\{0\}$. Assume that

(i) $x_{1}^{a_{1}}+\cdots+x_{n}^{a_{n}}=0$

(ii) $\sum_{i=1}^{n} \frac{1}{a_{i}} \leq \frac{1}{n-2}$

(iii) $x_{1}, \ldots, x_{n}$ are pairwise relatively prime in $B$.

Then $x_{1}, \ldots, x_{n} \in \mathrm{ML}(R)$ in each of the following cases:

(a) $R$ is a factorially closed subring of $B$ satisfying $x_{1}, \ldots, x_{n} \in R$;

(b) $R=B^{[N]}$ for some $N$.

Moreover, $x_{1}, \ldots, x_{n}$ belong to the rigid core of $B$.

Proof. (a) Let $R$ be a factorially closed subring of $B$ satisfying $x_{1}, \ldots, x_{n} \in R$. Let $D \in \operatorname{LND}(R)$ and let $A=\operatorname{ker}(D)$; we claim that $x_{1}, \ldots, x_{n} \in A$. To show this, we may assume that $D \neq 0$. Let $S=A \backslash\{0\}$, then $S^{-1} R=K^{[1]}$ where we set $K=\operatorname{Frac}(A)$. Note that $x_{1}, \ldots, x_{n}$ are pairwise relatively prime in $R$ by Remark 6.5(b), so they are pairwise relatively prime in $S^{-1} R=K^{[1]}$ by Remark 6.5(a). Then $x_{1}, \ldots, x_{n} \in K$ by Corollary 6.3. As $A$ is factorially closed in $R$, we have $R \cap K=A$ and hence $x_{1}, \ldots, x_{n} \in A$. This argument shows that $x_{1}, \ldots, x_{n} \in \operatorname{ML}(R)$, so case (a) is proved.

(b) Assume that $R=B^{[N]}$ for some $N$. Then $x_{1}, \ldots, x_{n}$ are pairwise relatively prime in $R$ by Remark 6.5(c). Applying part (a) to $x_{1}, \ldots, x_{n} \in R$ shows that $x_{1}, \ldots, x_{n} \in \operatorname{ML}\left(R^{\prime}\right)$ for any factorially closed subring $R^{\prime}$ of $R$ containing $x_{1}, \ldots, x_{n}$; in particular, $x_{1}, \ldots, x_{n} \in \operatorname{ML}(R)$. So we are done in case (b).

Observe that $x_{1}, \ldots, x_{n} \in \mathrm{ML}_{0}(B)$ and that if $i$ is such that $x_{1}, \ldots, x_{n} \in \mathrm{ML}_{i}(B)$ then $x_{1}, \ldots, x_{n} \in \mathrm{ML}_{i+1}(B)\left(R=\mathrm{ML}_{i}(B)\right.$ is a factorially closed subring of $B$, so case (a) gives $\left.x_{1}, \ldots, x_{n} \in \operatorname{ML}(R)\right)$. So $x_{1}, \ldots, x_{n}$ belong to the rigid core of $B$. 
One says that a ring $B$ of characteristic zero is stably rigid if $B \subseteq \operatorname{ML}(R)$ for every overring $R$ of $B$ such that $R=B^{[N]}$ for some $N$. In the following statement, we set $B_{a_{1}, \ldots, a_{n}}=$ $\mathbf{k}\left[X_{1}, \ldots, X_{n}\right] /\left\langle X_{1}^{a_{1}}+\cdots+X_{n}^{a_{n}}\right\rangle$ where $\mathbf{k}$ is a field of characteristic zero.

6.8. Corollary. Let $n \geq 3$ and $\left(a_{1}, \ldots, a_{n}\right) \in(\mathbb{N} \backslash\{0\})^{n}$ be such that $\sum_{i=1}^{n} \frac{1}{a_{i}} \leq \frac{1}{n-2}$. Then $B_{a_{1}, \ldots, a_{n}}$ is stably rigid.

Proof. The case $n=3$ is known (Theorem 7.1(b) of [10]), so we may assume that $n \geq 4$. Write $B=B_{a_{1}, \ldots, a_{n}}=\mathbf{k}\left[x_{1}, \ldots, x_{n}\right]$ where $x_{i}$ is the canonical image of $X_{i}$ in $B$. By Lemma $4.3, x_{1}, \ldots, x_{n}$ are prime elements of $B$ and are pairwise relatively prime in $B$. Consider an overring $R=B^{[N]}$ of $B$ for some $N$. Then $x_{1}, \ldots, x_{n} \in \operatorname{ML}(R)$ by case (b) of Theorem 6.7, so $B$ is stably rigid.

\section{REFERENCES}

[1] Ivan Cheltsov, Jihun Park, and Joonyeong Wong. Affine cones over smooth cubic surfaces. Journal of the European Mathematical Society, 18(7):1537-1564, 2016.

[2] Michael Chitayat, Locally nilpotent derivations and their quasi-extensions, Master's thesis, University of Ottawa, 2016.

[3] Daniel Daigle. Introduction to locally nilpotent derivations. Informal lecture notes prepared in 2010, available at http://aix1.uottawa.ca/ ddaigle

[4] Daniel Daigle. Tame and wild degree functions. Osaka Journal of Mathematics, 49:53-80, 2012.

[5] Daniel Daigle, Gene Freudenburg, and Lucy Moser-Jauslin. Locally nilpotent derivations of rings graded by an abelian group. In Algebraic Varieties and Automorphism Groups, volume 75 of Advanced Studies in Pure Mathematics, pages 29-48. Mathematical Society of Japan, 2017.

[6] Michiel de Bondt. Another generalization of Mason's ABC-Theorem. Preprint, arXiv:0707.0434v2, 2009.

[7] David Eisenbud. Commutative Algebra With a View Toward Algebraic Geometry. Springer-Verlag, New York, 1995. Graduate Texts in Mathematics, No. 150.

[8] Arno van den Essen. Polynomial automorphisms, volume 190 of Progress in Mathematics. Birkhäuser, 2000.

[9] David Finston and Stefan Maubach. Constructing (almost) rigid rings and a UFD having infinitely generated Derksen and Makar-Limanov invariant. Canadian Mathematics Bulletin, 53:77-86, 2010.

[10] Gene Freudenburg and Lucy Moser-Jauslin. Locally nilpotent derivations of rings with roots adjoined. Michigan Mathematical Journal, 62(2):227-258, 2013.

[11] Gene Freudenburg. Algebraic theory of locally nilpotent derivations, volume 136 of Encyclopaedia of Mathematical Sciences. Springer-Verlag, Berlin, second edition, 2017. Invariant Theory and Algebraic Transformation Groups, VII.

[12] Shulim Kaliman and Mikhail Zaidenberg. Miyanishi's characterization of the affine 3-space does not hold in higher dimensions. Annales de l'Institut Fourier (Grenoble), 50(6):1649-1669, 2000.

[13] José Seade. On the Topology of Isolated Singularities in Analytic Spaces, volume 241 of Progress in Mathematics. Birkhäuser Verlag, Basel, 2006.

Department of Mathematics and Statistics, University of Ottawa, Ottawa, Canada K1N 6N5

E-mail address: mchit007@uottawa.ca

E-mail address: ddaigle@uottawa.ca 